\title{
Disease activity, quality of life, and indirect costs of ulcerative colitis in Poland
}

\author{
Paweł Kawalec ${ }^{1}$, Ewa Stawowczyk ${ }^{2}$, Małgorzata Mossakowska ${ }^{3}$, Andrzej Pilc ${ }^{4}$ \\ ${ }^{1}$ Drug Management Department, Institute of Public Health, Faculty of Health Sciences, Medical College Jagiellonian University, \\ Krakow, Poland \\ ${ }^{2}$ StatSoft Polska Sp. z o.o., Krakow, Poland \\ ${ }^{3}$ Polish Association Supporting People with Inflammatory Bowel Disease "J-elita", Warsaw, Poland \\ ${ }^{4}$ Department of Neurobiology, Institute of Pharmacology, Polish Academy of Sciences, Krakow, Poland
}

Gastroenterology Rev 2017; 12 (1): 60-65

DOI: https://doi.org/10.5114/pg.2017.66324

Key words: ulcerative colitis, quality of life, indirect costs, productivity loss. Address for correspondence: Paweł Kawalec MD, PhD, Institute of Public Health, Medical College Jagiellonian University, 20 Grzegorzecka St,
31-531 Krakow, Poland, phone: +481242413 90, fax: +48 1242174 47, e-mail: pawel.kawalec@uj.edu.pl

\begin{abstract}
Introduction: Ulcerative colitis (UC) require expensive, lifelong treatment, which generates huge direct costs and has a significant impact on the quality of life, especially in the active state of the disease.

Aim: To assess the indirect costs, health-related quality of life, and clinical characteristics of patients with UC in Poland. Additionally, we investigated the association between activity of UC and productivity loss of patients in a Polish setting.

Material and methods: A questionnaire survey was conducted using the Patient Simple Clinical Colitis Activity Index (P-SCCAI) to assess disease activity, as well as the modified Work Productivity and Activity Impairment Questionnaire to assess productivity loss. The quality of life was presented as utility calculated with the EQ-5D-3L questionnaire. Indirect costs were assessed with the Human Capital Approach and were expressed in Polish zlotys (PLN) as well as in euros (€). Correlations were presented using the Spearman coefficient.

Results: We performed our analysis based on 202 full questionnaires collected. Mean patient age and age at disease onset were 33.14 years (standard deviation (SD): 9.90) and 26.35 years (SD: 8.89), respectively. The mean P-SCCAI score in the analysed group of patients was 8.26 , and the mean utility was 0.8651 . Average and median annual indirect costs per working person were $€ 2043$ and $€ 1389$ (8543 PLN and 5808 PLN), respectively, calculated using the gross domestic product, as well as $€ 4791$ and $€ 3257$ (20,026 PLN and 13,615 PLN), respectively, calculated using the gross value added. Total productivity loss was significantly correlated with the disease activity.

Conclusions: Ulcerative colitis causes a decrease in the quality of life as well as patients' productivity loss associated with both absenteeism and with presenteeism.
\end{abstract}

\section{Introduction}

Ulcerative colitis (UC) is an idiopathic inflammatory bowel disorder characterised by an inflammatory reaction involving the colonic mucosa [1, 2]. The clinical course is unpredictable and marked by alternating periods of exacerbations and remissions, which may occur spontaneously or in response to treatment changes or intercurrent illnesses [3, 4]. The prevalence of UC in Europe ranges from 4.9 to 505 per 100,000 people, while in North America it ranges from 37.5 to 248.6 per 100,000 people [5]. People affected with UC require expensive, lifelong treatment, which generates great direct costs to the national health insurance system. Additionally, it has a significant impact on the quality of life, especially in the active state of the disease. Ulcerative colitis is assumed to impose a considerable medical and societal burden, but there are no studies that would investigate both the quality of life and direct or indirect costs in a Polish setting. A recent review on that topic [6] showed that only 18 studies on the indirect costs of inflammatory bowel diseases in general were carried out worldwide, while only one was for UC.

The activity of UC is assessed with the Patient Simple Clinical Colitis Activity Index (P-SCCAI) [7], while the quality of life among patients with the disease can be 
measured using the EuroQol questionnaire five-dimension, three-level version (EQ-5D-3L).

The P-SCCAI is a patient-based translated version of the SCCAI questionnaire, which is used by clinicians to quantify disease activity of UC. The SCCAI refers to disease symptoms during the previous week. It consists of the following domains: bowel frequency during the day and during the night, urgency of defecation, blood in stool, general well-being, and a number of defined extracolonic features of UC. The clinician-based SCCAI is able to categorise two types of patients: those with inactive disease/remission (SCCAI score $<5$ ) and those with active disease (SCCAI score $\geq 5$ ) [7]. As in the case of the SCCAI questionnaire, the P-SCCAI refers to symptoms during the previous week. It was translated into language that is understandable for patients, and the medical terminology and disease symptoms were clarified. The interpretation of results is the same as in the SCCAI questionnaire [7].

The indirect costs that follow UC are associated with absence from work (absenteeism) and a decrease in the efficiency of work done by the sick patient (presenteeism). Absenteeism refers to the number of days on sick leave, periods of unemployment caused by a disease, and early leaving of the labour market due to sickness (e.g. early retirement or early death). Leaving the labour market due to early death is rather not calculated in the case of UC because there is no evidence that patients with UC have shorter life expectancy. Presenteeism refers to a situation when a sick person is present at work but his or her own productivity is lower than average due to the disease [8].

The Work Productivity and Activity Impairment (WPAI) questionnaire is a well-validated instrument to measure impairments in work and activities and is a standard analytic tool commonly used to assess absenteeism and presenteeism in a number of diseases [9]. Each category of indirect costs (absenteeism, presenteeism) can be calculated using two methods: the human capital approach (HCA) and the friction cost approach (FCA). The HCA converts the gross income that will not be earned in the future due to disease into real costs from a social perspective; hence it assumes that a decrease in productivity is permanent (irreplaceable). The FCA takes into account productivity losses until a new person is employed as a substitute for the sick one.

\section{Aim}

The aim of this study was to assess the indirect costs, health-related quality of life, and clinical characteristics of patients with UC measured by a UC disease activity index in Poland. The second aim was to investigate the association between disease activity and productivity loss of affected patients in a Polish setting.

\section{Material and methods}

A questionnaire-based survey was used to collect data on disease activity, health-related quality of life, and productivity loss of UC patients in Poland. The survey was started in October 2015 and has been planned to be finalised at the end of the first quartile of 2016. The results presented in this study are preliminary with a cut-off date of 31 December 2015, when a threshold population of the above 200 patients was reached. The study was performed in a group of patients with a diagnosis of UC, in cooperation with the Polish Association Supporting People with Inflammatory Bowel Disease "J-elita" (j-elita.org.pl). Patients filled in the paper version of the questionnaire regarding the disease activity index (P-SCCAI), comorbidities, prescribed treatment for UC, basic characteristics (age, sex, work status, date of disease onset, place of living, working status), disability status (EQ-5D-3L), expenses on medical and non-medical resources (e.g. private medical consultation, overthe-counter medication and dietary supplements, information materials) and loss of productivity (presenteeism, absenteeism), informal care, and benefits from a social care institution due to UC. Respondents were allowed to return the questionnaire by traditional mail, e-mail (scanned version), or saved directly on the server dedicated for this study (scanned version).

To assess the health-related quality of life, the EuroQol five-dimension, three-level version (EQ-5D-3L) questionnaire was used [10], and it was evaluated with the Polish norms presented by Golicki et al. [11]. The EQ-5D-3L takes into account five of the most important aspects of life: mobility, self-care, housekeeping activities, pain, and anxiety. For each domain the patient can report a lack of problems (represented by digit 1 ), the occurrence of some problems (digit 2), and the occurrence of many problems (digit 3). Data obtained using the EQ-5D-3L questionnaire can be converted into utility using population tariffs [8].

The WPAI questionnaire [10] was the starting point for the preparation of the part of the questionnaire concerning loss of productivity (presenteeism, absenteeism), usual activity impairment, informal care, and benefits from a social care institution; it was modified to adapt the questionnaire to part-time and seasonal employees, patients on rehabilitation benefit or other temporally inactive patients, or those awaiting the assessment of incapacity for work.

The HCA was used to estimate indirect costs due to absenteeism and presenteeism. Two macroeconomic indicators for Poland were considered: gross domestic product per capita (GDP, $€ 10,688$ or 44,677 PLN) and gross value added (GVA, €25,055 or 104,728 PLN) per worker presented in 2014 prices in euro (the ex- 
change rate used was 1 euro $=4.18$ PLN, which was the average exchange rate in 2015) with a correction factor of 0.65 (the conventional mean value of output elasticity of labour according to the Cobb-Douglas function of production; this approach is suggested by the European Commission because increasing the amount of a single factor of production, with all other factors of production constant, decreased the marginal output of a production) [12, 13]. The GDP per capita is a commonly used measure of a country's economic development. Its weakness is that it considers not only employees but also people who are economically inactive, such as children and retirees. It was calculated by dividing the GDP for Poland in the year 2014 $(1,719,097$ million PLN) by the Polish population in the same year $(38,478,600$; the above values come from the Central Statistical Office of Poland, www.stat.gov. $\mathrm{pl})$. The GVA per worker reflects true economic development in areas of production. It is expressed as value per worker and does not consider economically inactive people. The GVA per worker has been recognised as the most appropriate measurement for examining the decrease in human capital due to illness, as it represents the productivity of a country in all economic sectors. It was calculated by dividing the GVA for Poland in 2014 (1,525,193 million PLN) by the Polish population in the same year.

\section{Statistical analysis}

Continuous variables were summarised using means and standard deviations (SDs) or median and interquartile range (IQR), while nominal variables were summarised using counts and percentages. The Spearman's correlation was used to present the association between disease activity and absenteeism as well as presenteeism. $P$-values of less than 0.05 indicated statistical significance. Additionally, data on disease activity and generated indirect costs were presented on scatterplots. Absenteeism was presented as the number of days missed from work per year, while presenteeism was expressed as the percentage of standard work efficiency achieved due to UC. Statistical analyses were performed using Statistica ${ }^{\oplus}$.

\section{Results}

We obtained 202 completed questionnaires from patients aged from 18 to 71 years (average age was 33.14 years, SD: 9.90; median value was 31.00 years). The basic characteristics of the population are presented in Table I.

Of 202 patients, 190 (94.1\%) provided data on disease activity with the P-SCCAI questionnaire. Based on those values, the mean P-SCCAI score was 8.26 (SD: 5.26; range: 0-24). Among 190 patients with UC, 26.3\% had remission according to the P-SCCAI score. Patients also assessed disease activity on their own, based on the clinician's opinion during the last consultation. The compliance of patient's own assessment with the P-SCCAI score was $53.4 \%$.

Patients reported between 0 and 10 consultations with a clinician during the month before completing the questionnaire with a mean value of 1.22 (SD: 1.71), of which $62.12 \%$ were private visits with an average cost of the consultation of 141.69 PLN (SD: 59.99).

About $55.5 \%$ of patients spent from 0 to 100 PLN monthly on drugs prescribed by the clinician, and only $3.47 \%$ spent more than 600 PLN monthly. A similar amount of money was spent on dietary supplements and other medicines that are not prescribed by the clinician (over-the-counter drugs). The smallest amount of money was spent monthly on information materials about the disease: more than $90 \%$ of patients spent from 0 to 100 PLN, and no patient spent more than 400 PLN.

Almost $96 \%$ of patients (193 of 202) provided information about the drugs used. The vast majority of patients (92.23\%) took mesalazine. The other often administered drugs were: azathioprine (26.94\%), prednisone or prednisolone (21.24\%), and sulfasalazine (13.47\%). Only $4.66 \%$ of patients declared to use biologic drugs: adalimumab (2.59\%) and infliximab (2.07\%). Only 11 patients had surgery due to UC, which was restorative proctocolectomy in the majority of cases.

A number of UC patients (27.8\%) required help with daily activities; in $95.5 \%$ of cases, the help was provided by family members. Only $11.2 \%$ of patients received financial benefits from state institutions in the form of disability pension, social pension, illness or rehabilita-

Table I. The basic characteristics of the population considered in the study

\begin{tabular}{|c|c|c|c|}
\hline \multicolumn{2}{|l|}{ Characteristic } & $N$ & Value \\
\hline \multicolumn{2}{|l|}{ Age [years] } & 202 & 33.14 (SD: 9.90), range: $18-71$ \\
\hline \multicolumn{2}{|l|}{ Male } & 198 & $75(37.88 \%)$ \\
\hline \multicolumn{2}{|c|}{ Disease onset [years] } & 201 & 26.35 (SD: 8.89), range: 8-62 \\
\hline \multirow[t]{3}{*}{ Place of living } & City $<100,000$ citizens & \multirow[t]{3}{*}{200} & $60(30.00 \%)$ \\
\hline & City $\geq 100,000$ citizens & & $119(59.50 \%)$ \\
\hline & Village & & $21(10.50 \%)$ \\
\hline
\end{tabular}


tion benefit, and $8.4 \%$ received financial support from other sources.

Data on employment status was collected for 202 patients, of which 150 (74.26\%) were currently working. Among all working patients, 130 were on full-time contract. Thirty-four (16.83\%) patients were still studying and $6.44 \%$ did not have paid employment. Only 7 patients were on pension due to UC and seven were unable to work. The average monthly amount of social pension was 605.75 PLN, and it was slightly lower than pension due to inability to work (681.25 PLN).

The average annual number of days off work due to illness was 30.49 (SD: 65.37; range: 0-279). Most people $(65.31 \%)$ reported lack of days off work due to the disease.

The relationship between absenteeism and disease activity (moderate correlation of 0.4603 with a $p$-value of less than 0.05) is presented in Figure 1.

Average on-the-job productivity loss was $19.38 \%$ (SD: $23.64 \%$; range: $0-100 \%)$, which represents the extent of presenteeism. Only $4.1 \%$ of working patients reported on-the-job productivity loss of at least $70 \%$, while $44.8 \%$ of working patients reported no on-the-job productivity loss (value 0). Data were presented for 145 working patients with UC; five working patients did not provide data on on-the-job productivity loss.

The relationship between presenteeism and disease activity (moderate correlation of 0.4194 with a $p$-value of less than 0.05 , which indicates statistical significance) is presented in Figure 2. Loss of on-thejob productivity was positively correlated with disease activity.

Using the GDP per capita, the indirect costs of absenteeism per working person were $€ 679$ (SD: $€ 1691)$ or 2837 PLN (SD: 7070 PLN), and the indirect costs of presenteeism per working person were 1346 (SD: €1642; median: €695, IQR: €1642) or 5628 PLN

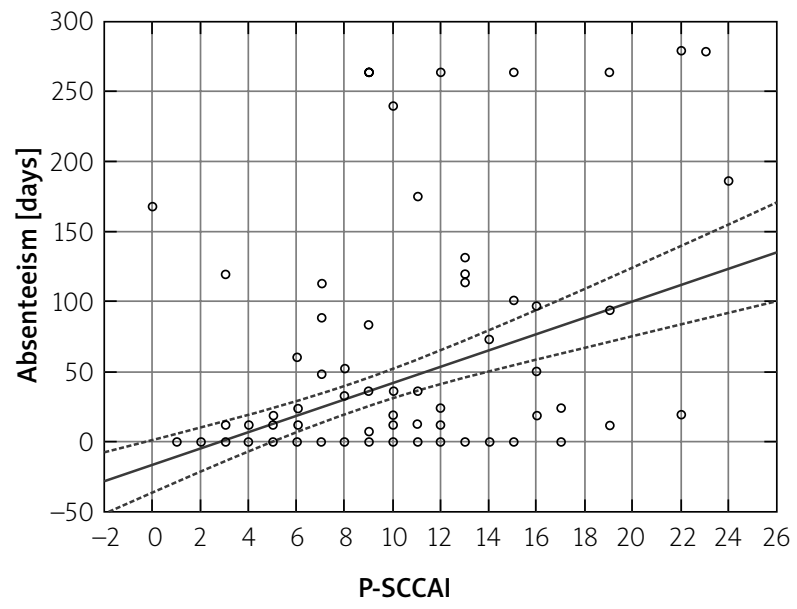

Figure 1. Relationship between absenteeism and disease activity
(SD: 6864 PLN; median: 2904 PLN, IQR: 6864 PLN). Taking into account the GVA per worker, the costs were as follows: €1591 (SD: €3965) or 6651 PLN (SD: 16,573 PLN) for absenteeism, and €3156 (SD: €3849; median: $€ 1629$, IQR: $€ 3849$ ) or 13,192 PLN (SD: 16,089 PLN; median: 6807 PLN, IQR: 16,089 PLN) for presenteeism.

The average annual indirect cost of both absenteeism and presenteeism per working person was $€ 2043$ (SD: 2876; median: €1389, IQR: 3215) or 8543 PLN (SD: 12,020; median: 5808 PLN, IQR: 13,441) calculated using the GDP and €4791 (SD: 6,741; median: €3257, IQR: 7537) or 20,026 PLN (SD: 28,178; median: 13,615 PLN, IQR: 31,507$)$ calculated using the GVA. Data were based on the answers of 144 working patients with UC. The cost of presenteeism accounted for $65.87 \%$ of the total indirect costs.

As a sensitivity analysis, the results without the correction factor (marginal productivity of labour) were presented. Total annual indirect costs per patient were $€ 2989$ (SD: €3801) or 12,495 PLN (SD: 15,887 PLN), as calculated using the GDP, and €7007 (SD: €8910) or 29,289 PLN (SD: 37,242 PLN), as calculated using the GVA.

The relationship between indirect costs calculated using the GDP and disease activity (moderate correlation of 0.4651 with $p$-value of less than 0.05 ) is presented in Figure 3. The correlation of disease activity with indirect costs calculated using the GVA was the same.

Data on the quality of life were also reported by almost all respondents (201 of 202; 99.5\%). Based on data from Table II, the health states were created. The most common state was " 11122 ", reported by 58 (28.86\%) patients. The highest (worst) state observed was "22222" and occurred in 3 (1.49\%) patients. Based on these data the utility was calculated using the Polish tariff [14]. The mean utility equalled 0.8651 (SD: 0.1285; range: $0.1720-1$ ).

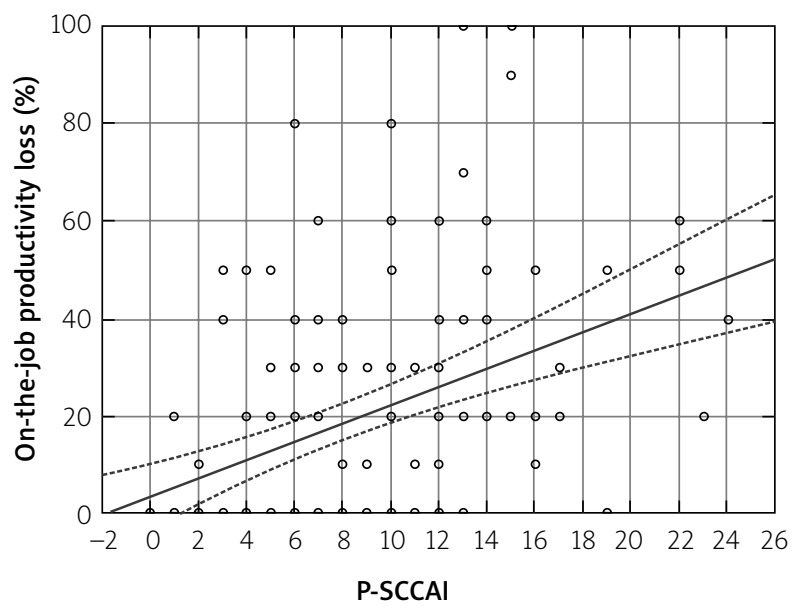

Figure 2. Relationship between presenteeism and disease activity 


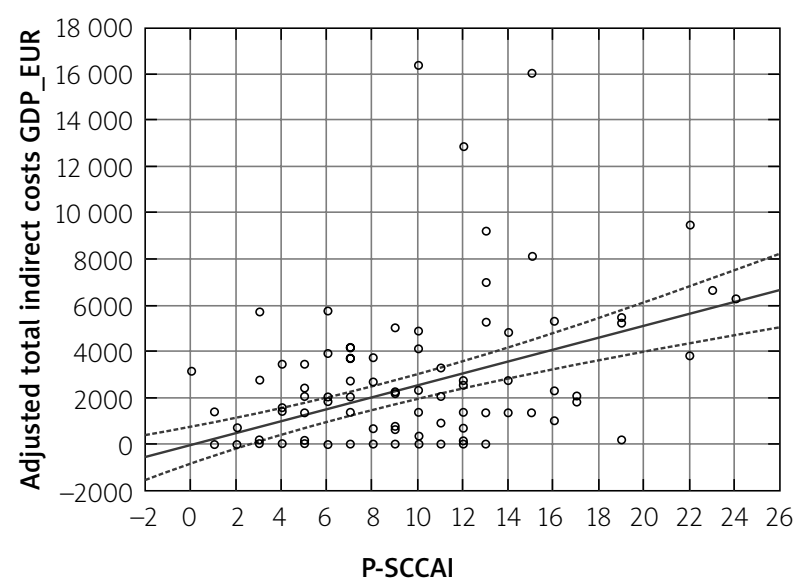

Figure 3. Relationship between adjusted total indirect costs and disease activity

\section{Discussion}

This study examined the impact of UC on the work productivity of affected patients. Despite the fact that UC is known to impose a high clinical and societal burden, there is no evidence to confirm this in Poland. Our study proved that UC reduces the quality of life, especially in patients with active disease. The study also showed that the greater the disease activity, the lower the quality of life. Moreover, UC generates significant indirect costs, mainly due to presenteeism. We observed a significant moderate correlation between disease activity and indirect costs.

There have been no previous attempts to assess the indirect costs of UC in Poland. Therefore, it seems necessary to assess the societal burden of the disease on a representative group of patients in Poland, using a comprehensive approach. Additionally, the available data suggest that there may be significant between-country differences in health-related quality of life of the same population, and the differences may even lead to changing the conclusions of economic evaluations [15]. In the absence of reliable information on the health-related quality of life of UC in Poland, there was a need to conduct such an assessment.

Our project involved the assessment of the indirect costs of both absenteeism and presenteeism, based on our own survey questionnaire. The methodology used and the results obtained provide novel data that fill the information gap. However, our study has several limitations, and the results are preliminary and should be interpreted with caution. They may be used as the basis for further, more sophisticated studies.

The major limitation is the recruitment of patients. The sample size was reasonably good, but the methods for the inclusion of respondents without randomised allocation provided results that are not fully representative of the whole UC population. In the analysed group of patients there was a slight overrepresentation of women; however, the observed difference in sex structure for UC proved insignificant. We also recorded a high percentage of individuals who live in big cities (with 100,000 citizens or more) and a small percentage of village citizens, which might have influenced labour market activity and might have affected our results. Data on disease activity were provided by patients, not by clinicians, which may be another limitation. However, the agreement between self- assessment by patients and the P-SCCAI score was quite good and equalled $53.4 \%$.

In order to compare our results with findings of other investigators, we performed a review of medical databases. We discovered no studies considering the indirect costs of UC in Poland. However, we identified a systematic review performed by Kawalec and Malinowski (2015) [6], indicating that only 18 studies on the indirect costs of inflammatory bowel diseases were conducted worldwide: six studies on Crohn's disease (CD), only one for UC, and 11 on inflammatory bowel diseases in general. The identified study concerning UC [16] (available as an abstract) considered only lost wages from time away from work due to disability and medically-related absenteeism. Total annual indirect costs were calculated to be 4087 USD (2010 values; $€ 4070$ in 2015 values). It is difficult to compare our results with the results of the above study [16] because it was performed in a different setting and it concerned only privately insured U.S. employees.

One study was additionally identified concerning the indirect costs of selected inflammatory diseases in Poland, the 'Move to Work' or M2W study [17]. The aim

Table II. Quality of life assessment in the considered population

\begin{tabular}{|c|c|c|c|}
\hline \multirow[t]{2}{*}{ Life domain } & \multicolumn{3}{|c|}{ Problems reported with specific life domains } \\
\hline & No & Some & A lot \\
\hline Mobility & $179(89.05 \%)$ & $22(10.95 \%)$ & $0(0 \%)$ \\
\hline Self-care & 197 (98.01\%) & $4(1.99 \%)$ & $0(0 \%)$ \\
\hline Usual activities & $149(74.13 \%)$ & $49(24.38 \%)$ & $3(1.49 \%)$ \\
\hline Pain & $69(34.33 \%)$ & $127(63.18 \%)$ & $5(2.49 \%)$ \\
\hline Anxiety & $67(33.33 \%)$ & $124(61.69 \%)$ & $10(4.98 \%)$ \\
\hline
\end{tabular}


of this study was to assess the indirect costs of rheumatoid arthritis, psoriasis and Crohn disease (CD) in Poland. The study involved 2100 patients from Poland, including 464 with CD. The results of the study indicated that the highest indirect cost associated with absenteeism was among patients with rheumatoid arthritis, and the lowest was among patients with psoriasis. It was found that the lowest cost associated with presenteeism was generated by patients with CD. The annual indirect cost per working patient with $C D$ was estimated at 10,414 PLN (absenteeism: €2491) and 13,187 (presenteeism: €3155). The M2W study included only patients undergoing outpatient visits (inpatient care was excluded), and in contrast to other illnesses, more than $60 \%$ of patients with CD were in remission or the disease had very low activity. Narrowing the included population resulted in the study being unrepresentative.

\section{Conclusions}

People affected with UC require expensive, lifelong treatment, which constitutes a great burden to the national health insurance system. The UC generates considerable indirect costs, mainly due to lower productivity at work, but also due to absenteeism. The greater the disease activity, the greater the indirect costs generated by the disease. The UC causes a significant decrease in patient's quality of life.

\section{Acknowledgments}

The study was partially funded by a scientific grant (Jagiellonian University Medical College; statutory project Drug Management Department, Institute of Public Health JU MC). None of the authors has any conflict of interest to declare that refers to this study. All listed authors approved the final version of the manuscript.

PK and ES conceived the conception and design of the study, including protocol and questionnaires preparation. MM provided critical input and revised the questionnaires. PK, ES and MM contributed in acquisition of data. PK and ES carried out the data management, statistical analysis, interpretation of data and prepared the draft of the manuscript. All authors contributed to editing the manuscript and approved the final version submitted for publication. PK is the guarantor.

\section{Conflict of interest}

The authors declare that they have no conflict of interest.

\section{References}

1. Baumgart DC, Sandborn WJ. Inflammatory bowel disease: clinical aspects and established and evolving therapies. Lancet 2007; 369: 1641-57.
2. Feagan BG, Reinisch W, Rutgeerts P, et al. The effects of infliximab therapy on health-related quality of life in ulcerative colitis patients. Am J Gastroenterol 2007; 102: 794-802.

3. Kornbluth A, Sachar DB. The Practice Parameters Committee of the American College of Gastroenterology. Ulcerative Colitis Practice Guidelines in Adults: American College of Gastroenterology, Practice Parameters Committee. Am J Gastroenterol 2010; 105: 501-23.

4. Ordás I, Eckmann L, Talamini M, et al. Ulcerative colitis. Lancet 2012; 380: 1606-19.

5. Tontini GE, Vecchi M, Pastorelli L, et al. Differential diagnosis in inflammatory bowel disease colitis: state of the art and future perspectives. World J Gastroenterol 2015; 21: 21-46.

6. Kawalec P, Malinowski KP. Indirect health costs in ulcerative colitis and Crohn's disease: a systematic review and meta-analysis. Expert Rev Pharmacoecon Outcomes Res 2015; 15: 253-66.

7. Evertsz FB, Nieuwkerk PT, Stokkers PCF, et al. The Patient Simple Clinical Colitis Activity Index (P-SCCAI) can detect ulcerative colitis (UC) disease activity in remission: a comparison of the P-SCCAI with clinician-based SCCAI and biological markers. J Crohns Colitis 2013; 7: 890-900.

8. Krol M, Brouwer W. How to estimate productivity costs in economic evaluations. Pharmacoeconomics 2014; 32: 335-44.

9. Reilly MC, Zbrozek AS, Dukes EM. The validity and reproducibility of a work productivity and activity impairment instrument. Pharmacoeconomics 1993; 4: 353-65.

10. EuroQol. http://www.euroqol.org/ Last Access: October 2015.

11. Golicki D, Jakubczyk M, Niewada M, et al. Valuation of EQ-5D health states in Poland: first TTO based social value set in Central and Eastern Europe. Value Health 2010; 13: 289-97.

12. Central Statistical Office of Poland, http://stat.gov.pl/en/ Last access: January 2016

13. Havik K, Mc Morrow K, Orlandi F, et al. The production function methodology for calculating potential growth rates and output gaps. Economic Papers 535, November 2014, http://ec.europa. eu/economy_finance/publications/economic_paper/2014/pdf/ ecp535_en.pdf.

14. Bouwmans C, De Jong K, Timman R, et al. Feasibility, reliability and validity of a questionnaire on healthcare consumption and productivity loss in patients with a psychiatric disorder (TiC-P). BMC Health Serv Res 2013; 13: 217.

15. Noyes K, Dick AW, Holloway RG. The implications of using US-specific EQ-5D preference weights for cost-effectiveness evaluation. Med Decis Making 2007; 27: 327-34.

16. Cohen R, Rizzo J, Yang M, et al. Direct and indirect utilization and costs associated with ulcerative colitis. Am J Gastroenterol 2012; 107: S608-95.

17. CEESTAHC. Przewlekłe choroby zapalne mediowane immunologicznie - ocena kosztów pośrednich w Polsce. Kraków, January 2014. On-line access; http://m2w.ceestahc.org/media/ M2W_Koszty_posrednie_IMIDs_W_Polsce_podsumowanie. pdf (March 15, 2016).

Received: 4.04.2016

Accepted: 10.05 .2016 